\title{
Polymer-Assisted Isolation of Single Wall Carbon Nanotubes in Organic Solvents for Optical-Quality Nanotube-Polymer Composites
}

\author{
T. Hasan, ${ }^{*, \dagger}$ P. H. Tan, ${ }^{\dagger, *}$ F. Bonaccorso, ${ }^{\dagger}, \$$ A. G. Rozhin, ${ }^{\dagger}$ V. Scardaci, ${ }^{\dagger}$ W. I. Milne, ${ }^{\dagger}$ and \\ A. C. Ferrari* ${ }^{\dagger}$ \\ Engineering Department, Cambridge University, 9 JJ Thomson Avenue, Cambridge CB3 OFA, U.K., State Key \\ Laboratory for Superlattices and Microstructures, P.O. Box 912, Beijing 100083, China, and CNR-IPCF and \\ Dipartimento di Fisica della Materia e Ingegneria Elettronica, Università di Messina, Messina, Italy
}

Received: August 6, 2008; Revised Manuscript Received: October 12, 2008

\begin{abstract}
Dispersions of isolated single wall carbon nanotubes (SWNTs) in pure organic solvents can usually be prepared only at low concentrations $(\sim 0.01-0.02 \mathrm{~g} / \mathrm{L})$. Here, we use poly(vinylpyrrolidone) as a dispersant for unfunctionalized SWNTs in $N$-methyl-2-pyrrolidone and achieve concentrations of up to $\sim 0.16 \mathrm{~g} / \mathrm{L}$. Such dispersions remain stable by visual inspection for at least 1 year after preparation. Well-resolved features in the absorption spectra are observed, implying the presence of isolated SWNTs or small bundles. This is further confirmed by photoluminescence excitation spectroscopy. Dispersions enriched with isolated SWNTs are obtained by ultracentrifugation. Strong photoluminescence signal from nanotubes with $\sim 1$ nm diameter indicates the dispersion mechanism to be diameter selective. SWNT-polymer composites prepared from filtered dispersions, with styrene methyl methacrylate (SMMA) as host matrix, are obtained with sub-micrometer aggregations and high optical density. The high transparency of SMMA below $1650 \mathrm{~nm}$ is promising when such SWNT-polymer composites are to be used in telecommunication applications.
\end{abstract}

\section{Introduction}

Single wall carbon nanotubes (SWNTs) form bundles ${ }^{1-3}$ due to van der Waals forces. ${ }^{3-5}$ A most effective way to untangle them is through mechanical ${ }^{6-13}$ or chemical ${ }^{14-17}$ means in a liquid environment. The former method, generally performed by ultrasonic treatments, relies on physical adsorption of solvent or dispersant molecules on the nanotube surface, which prevent their reaggregation. ${ }^{6,7,10,12}$ Unlike covalent functionalization, this adsorption process does not disrupt the extended $\pi$-network and, hence, causes little or no change in their electronic properties. ${ }^{18-20}$ For applications exploiting their pristine electronic structure, dispersion of unfunctionalized SWNTs is therefore the preferred route. Furthermore, isolated dispersions of SWNTs in various solvents are ideal for fundamental studies and to assess their interaction with surrounding dielectric environments. ${ }^{21-26}$

SWNTs offer an excellent alternative to conventional ultrafast all-optical switches, as they exhibit ultrafast recovery time $(\sim 1$ ps) with high third-order nonlinear polarizability. ${ }^{27-29}$ For such applications, they must be incorporated in small submicrometer bundles in optically transparent polymer matrices to avoid unwanted optical losses ${ }^{30}$ and overtone absorption at the device operation wavelength. ${ }^{31}$ High optical damage threshold and possibility of using SWNT-polymer composites in both transmissive and reflective configurations are attractive in realizing other optical devices, e.g., noise suppressors, signal regenerators, waveguides, etc. ${ }^{28,32}$ Another desirable property of the host polymer matrices for communication devices is the stability of their optical properties against humidity, ${ }^{31}$ i.e., the polymers must be water insoluble. Therefore, for such applica-

* To whom correspondence should be addressed. Phone: +44 12237 48351. Fax: +44 12237 48348. E-mail: acf26@eng.cam.ac.uk.

Cambridge University.

\$ State Key Laboratory for Superlattices and Microstructures.

$\S$ CNR-IPCF and Dipartimento di Fisica della Materia e Ingegneria Elettronica, Università di Messina. tions, dispersion of SWNTs in organic solvents is important in order to realize humidity resistant SWNT-polymer composites, such as poly(methyl methacrylate) (PMMA), styrene methyl methacrylate (SMMA), polycarbonate (PC), to name a few.

It is possible to prepare stable dispersions of unfunctionalized SWNTs in pure organic solvents, but only in low concentrations. ${ }^{11,33-35}$ Higher concentrations usually lead to reaggregation in the absence of dispersant molecules. ${ }^{11,12}$ To increase the SWNT loading in organic solvents, polymers such as polyimide and PMMA have been previously used through noncovalent interaction. ${ }^{36-38}$ Recently, we have shown that SWNTs dispersed in pure $N$-methyl-2-pyrrolidone (NMP) can be stabilized and spontaneously 'debundled' by simple addition of poly(vinylpyrrolidone) (PVP), a linear polymer. ${ }^{11,12}$ Here, we show that PVP can also be used to disperse SWNTs with concentrations of up to $\sim 0.16 \mathrm{~g} / \mathrm{L}$. Photoluminescence excitation (PLE) spectroscopy confirms the presence of small SWNT bundles. The dispersions remain stable for at least one year after preparation, but reaggregate if the PVP concentration is reduced by the addition of pure NMP. We then prepare SWNT-SMMA composites from the filtered dispersions. Optical inspection reveals no aggregation of SWNTs, making them ideal for communication applications. ${ }^{30}$ Mostly isolated SWNTs are obtained by ultracentrifugation at 300,000g. Photoluminescence (PL) from such dispersions indicates that the PVP-aided dispersion mechanism is diameter selective. Weak optical signatures of exciton energy transfer (EET) between nanotubes in small SWNT bundles are also observed.

\section{Experimental Section}

Dispersions are prepared by adding $\sim 0.35 \mathrm{~g} / \mathrm{L}$ of purified HiPco $^{39}$ SWNTs (lot no. PO279, Carbon Nanotechnologies, Inc.) and $0-70 \mathrm{~g} / \mathrm{L}$ of PVP (Sigma-Aldrich; average molecular weight $(\mathrm{av} M W) \approx 29 \mathrm{kDa}$ ) to $10 \mathrm{~mL}$ spectroscopic grade NMP 
TABLE 1: Composition of SWNT Dispersions Discussed in This Article

\begin{tabular}{|c|c|c|c|}
\hline sample & dispersant $(\mathrm{g} / \mathrm{L})$ & $\begin{array}{l}\text { final SWNT } \\
\text { concentration }(\mathrm{g} / \mathrm{L})^{a}\end{array}$ & comments \\
\hline$A$ & PVP (14) & 0.11 & Sonicated and filtered. \\
\hline A1 & PVP & & Sonicated, filtered and centrifuged. \\
\hline$B$ & Igepal DM-970 (3.5) & 0.022 & Sonicated and filtered. \\
\hline$C$ & Pluronic F98 (6.27) & 0.010 & Sonicated and filtered. \\
\hline$D$ & none & $0.010-0.020^{b}$ & Dispersed in pure NMP. Sonicated and filtered. \\
\hline series $X$ & PVP (see comments) & See Figure 2 & $\begin{array}{l}\text { Sonicated and filtered. PVP concentration } 0,1.75,3.5,7,10.5,14 \text {, } \\
21,35,52.5 \text {, and } 70 \mathrm{~g} / \mathrm{L} \text {. }\end{array}$ \\
\hline series $Y$ & PVP (see comments) & $12.5 \%$ of series $X$ & Same as above \\
\hline series $Z$ & PVP (see comments) & $12.5 \%$ of series $X$ & $\begin{array}{l}\text { Sonicated and filtered. PVP concentration } 0,0.22,0.44,0.88,1.31 \text {, } \\
1.75,2.63,4.38,6.56 \text {, and } 8.75 \mathrm{~g} / \mathrm{L} \text {. }\end{array}$ \\
\hline
\end{tabular}

${ }^{a}$ Concentrations exclude $20 \mathrm{wt} \%$ impurities present in the purified HiPco SWNTs. ${ }^{11}{ }^{b} \mathrm{SWNT}_{\text {conc }}$ in pure NMP usually varies between 0.01 and $0.02 \mathrm{~g} / \mathrm{L}$ with $\sim 0.03 \mathrm{~g} / \mathrm{L}$ starting $\mathrm{SWNT}_{\text {conc }}$.

(Sigma-Aldrich). The mixtures are ultrasonically treated at 20 $\mathrm{kHz}$ for $3 \mathrm{~h}$ at $10-12{ }^{\circ} \mathrm{C}$ (Bioruptor; Diagenode). Then they are immediately vacuum-filtered by binder-free glass fiber filters (Millipore; $0.7 \mu \mathrm{m}$ average retention). Using this method, sample $A$ is prepared with $14 \mathrm{~g} / \mathrm{L}$ PVP. An aliquot of this sample is ultracentrifuged at $300000 \mathrm{~g}$ using a MLA-130 fixed-angle rotor (Beckman) for $2 \mathrm{~h}$ to eliminate bundles. The top $30 \%$ of the ultracentrifuged dispersion is then decanted (sample $A l$ ) for characterization. We also prepare vacuum-filtered SWNT dispersions with two nonionic surfactants (samples $B$ and $C$ ) in identical conditions. In this case, Pluronic F98 (BASF; av MW $\approx 13 \mathrm{kDa}$ ) and Igepal DM-970 (Sigma-Aldrich; av MW $\approx 7.2$ $\mathrm{kDa}$ ) aided dispersions with the same molar concentration as PVP $(\sim 0.48 \mathrm{mM})$ are prepared. A SWNT dispersion in pure NMP serves to demonstrate the effect of dielectric screening due to dispersant molecules ${ }^{8,9,11,24}$ (sample $D$ ). In addition, another set (series $X$ ) with a range of PVP concentrations $(0-70$ $\mathrm{g} / \mathrm{L})$ in NMP is prepared. In series $Y, 7$ parts of NMP with appropriate concentration of PVP are added to 1 part of corresponding dispersion from series $X$. For series $Z, 7$ parts of only pure NMP are added to 1 part of corresponding dispersion from series $X$. Table 1 lists all the dispersions.

SWNT-polymer composites are prepared by mixing and ultrasonicating dissolved SMMA in NMP with the vacuum filtered SWNT dispersions. The solvent is then slowly evaporated at $80{ }^{\circ} \mathrm{C}$, leaving SWNTs incorporated in SMMA. Absorption spectra are measured with a Perkin-Elmer Lambda 950 spectrophotometer with $0.4 \mathrm{~nm}$ steps and $10 \mathrm{~mm}$ optical path. These are background subtracted to account for solvent and dispersants. Dispersions with high SWNT loading are diluted to avoid scattering. A JY Fluorolog-3, equipped with a liquid $\mathrm{N}_{2}$ cooled InGaAs detector is used for PLE measurements by scanning the excitation wavelength from 560 to $800 \mathrm{~nm}$ with $5 \mathrm{~nm}$ steps, 180 s exposure, and a $950-1500 \mathrm{~nm}$ emission range.

\section{Results and Discussions}

3.1. Stability and Loading of Dispersed Nanotubes. The Beer-Lambert law is used to estimate SWNT concentration $\left(\mathrm{SWNT}_{\text {conc }}\right)$. Absorption coefficients $\left(\alpha_{\lambda}\right)$ of SWNTs in NMP at four well-defined SWNT absorption bands (506, 660, 871, and $1308 \mathrm{~nm}$ ) are measured from a set of SWNT dispersions of known concentration. ${ }^{11}$ The $\alpha_{\lambda}$ values thus obtained are then used to find the average $\mathrm{SWNT}_{\text {conc }}$, as reported in Table 1. Figure 1 shows the absorption spectra of the as-prepared samples $A$, $B$, and $C$. Here, resolved spectral features of interband excitonic transitions demonstrate good dispersion even without ultracentrifuge treatment. The $\sim 1430 \mathrm{~nm}$ band, indicated by the vertical solid line, is mainly due to the $\mathrm{O}-\mathrm{H}$ vibration overtones from

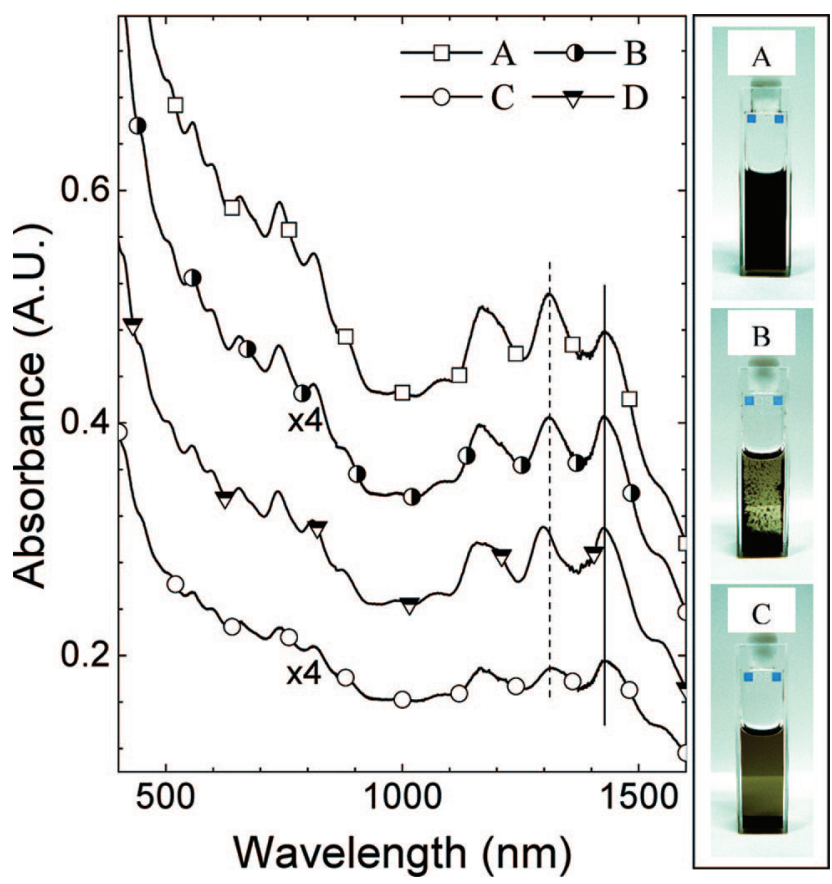

Figure 1. Absorption spectra of SWNTs freshly dispersed in NMP. Background of solvent and dispersants has been subtracted where appropriate. Samples $A, B$, and $C$ are diluted before measurement to avoid scattering. The $\sim 1430 \mathrm{~nm}$ absorption band (vertical solid line) is due to an $\mathrm{O}-\mathrm{H}$ vibration overtone ${ }^{40}$ The vertical dashed line at $\sim 1300 \mathrm{~nm}$ indicates the shift in SWNT transition energies due to dielectric screening. Photographic images of samples $A, B$, and $C$ show the original dispersions after $\sim 1$ year incubation.

moisture present in the sample. ${ }^{31,40}$ With equivalent molar concentration of the dispersants $(\sim 0.48 \mathrm{mM})$ and $0.35 \mathrm{~g} / \mathrm{L}$ of starting $\mathrm{SWNT}_{\text {conc }}, \mathrm{PVP}$ disperses $\sim 5$ and $\sim 10$ times more SWNTs than Igepal DM-970 (sample $B$ ) and Pluronic F98 (sample $C$ ), respectively. Compared to sample $A$, a minor blueshift $(\sim 0.5 \mathrm{meV}$ at about $1 \mathrm{eV})$ in the absorption spectrum of sample $B$ is observed. On the other hand, sample $C$ shows a red-shift of $\sim 3.5 \mathrm{meV}$ at about $1 \mathrm{eV}$ compared to sample $A$. We attribute these shifts in optical transition energy to dielectric screening; either due to the dispersant molecules surrounding the SWNTs ${ }^{8,9,11,24}$ or to the presence of SWNT bundles. ${ }^{41-44}$ Also, sample $A$ exhibits a red-shift of $\sim 9 \mathrm{meV}$ (at about $1 \mathrm{eV}$ ) compared to sample $D$ due to dielectric screening by the PVP molecules. ${ }^{11,12}$ The photographs of the same samples after 1 year incubation are also presented in Figure 1, illustrating the longterm instability of samples $B$ and $C$. In addition to the surfactant chemistry, the difference in the degree of aggregate formation in these two samples is likely to depend on $\mathrm{SWNT}_{\text {conc. Higher }}$ 


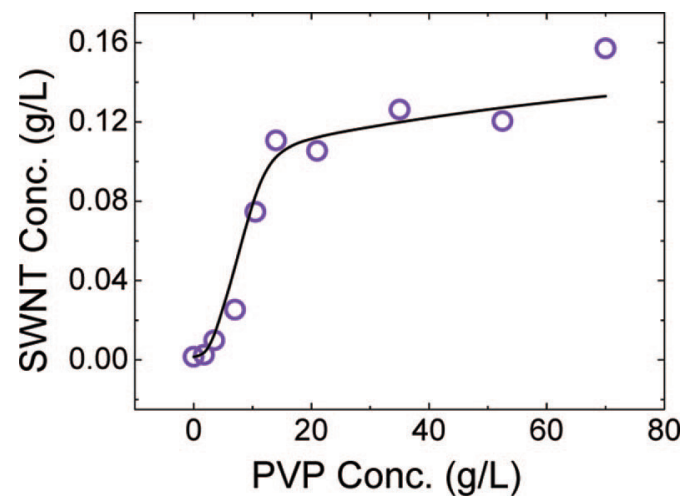

Figure 2. SWNT concentration in the filtered dispersions as a function of PVP concentration. The solid line is as a guide to the eye.

concentration (i.e., shorter mean distance between dispersed SWNTs) results in more aggregations. Disregarding dispersant effects on solution-stabilization, sample $A$, with higher $\mathrm{SWNT}_{\text {conc }}$ than samples $B$ and $C$, is thus expected to form aggregates quicker than the latter samples. However, it remains very stable, demonstrating that PVP effectively prevents formation of large SWNT aggregates. Recently, we reported that PVP-induced stabilization of SWNTs in NMP is not thermodynamically stable though it greatly decelerates nanotube reaggregation. ${ }^{11,12} \mathrm{We}$ observe no PL from SWNTs dispersed in NMP-PVP a year after preparation. This implies gradual formation of large SWNT bundles.

$\mathrm{SWNT}_{\text {conc }}$ in the dispersions can be increased by tuning the PVP concentration $\left(\mathrm{PVP}_{\text {conc }}\right)$. Figure 2 shows $\mathrm{SWNT}_{\text {conc }}$ in the filtered dispersions for a range of $\mathrm{PVP}_{\text {conc }}$. For $\mathrm{PVP}_{\text {conc }}$ of 14 $\mathrm{g} / \mathrm{L}$ or above, $\mathrm{SWNT}_{\text {conc }}$ in the dispersions averages $\sim 0.12 \mathrm{~g} / \mathrm{L}$ without aggregation. Hence, at least $14 \mathrm{~g} / \mathrm{L}(\sim 0.5 \mathrm{mM}) \mathrm{PVP}_{\text {conc }}$ is needed to disperse $\sim 35 \%$ of the initial ( $\sim 0.35 \mathrm{~g} / \mathrm{L})$ SWNTs. If we consider that the average length of SWNTs is $\sim 500 \mathrm{~nm}$ after the ultrasonication process, then an $(8,6)$ SWNT will weight $\sim 700 \mathrm{kDa}$. This corresponds to $\sim 3000$ PVP molecules per SWNT in the above-mentioned case. We obtain lower $\mathrm{SWNT}_{\text {conc }}$ with the surfactants (Table 1). In addition, these surfactant-aided SWNT dispersions show signs of aggregations within weeks of preparation. Note that the initial $\mathrm{SWNT}_{\text {conc }}$ is an important parameter for efficient SWNT dispersion and stabilization. For a high initial $\mathrm{SWNT}_{\text {conc }}$, the nanotubes tend to form large aggregates immediately after the ultrasonic treatment, making the resulting dispersion difficult to filter. This, in turn, greatly reduces the total amount of SWNTs in the filtered dispersion. For example, $\mathrm{SWNT}_{\text {conc }}$ of $0.033 \mathrm{mg} / \mathrm{L}$ in pure NMP can be easily obtained with a low initial $\mathrm{SWNT}_{\text {conc }}(\sim 0.08 \mathrm{~g} / \mathrm{L}),{ }^{11}$ but similar ultrasonic treatment with $0.35 \mathrm{~g} / \mathrm{L}$ initial $\mathrm{SWNT}_{\text {conc }}$ yields only $\sim 0.0015 \mathrm{~g} / \mathrm{L}$ SWNTs. For $70 \mathrm{~g} / \mathrm{L} \mathrm{PVP}_{\text {conc, }}$, we obtain $\mathrm{SWNT}_{\text {conc }} \approx 0.16 \mathrm{~g} / \mathrm{L}$, dispersing nearly $45 \%$ of initial SWNTs. Such loading of unfunctionalized SWNTs is an improvement of 1 order of magnitude compared to pure NMP..$^{1,33,34}$ The trend in Figure 2 indicates that even higher $\mathrm{SWNT}_{\text {conc }}$ could be achieved with further optimization.

In addition to the improvement of short-term stability of SWNT dispersions in NMP ${ }^{11}$ PVP improves their long-term ( $\sim 1$ year) stability. This is demonstrated in Figure 3(i)-(iii). When $\mathrm{PVP}_{\text {conc }}$ is kept constant (series $X$ and $Y$ ) at room temperature $\left(\sim 21{ }^{\circ} \mathrm{C}\right)$, the dispersions remain free from aggregates. However, the dispersions in series $Y$ with less than $\sim 2 \mathrm{~g} / \mathrm{L}$ PVP $(\sim 0.07 \mathrm{mM})$ form miniscule aggregates. SWNT aggregation, but on a larger scale, is observed in series $Z$ with lower $\mathrm{PVP}_{\text {conc }}$ than the corresponding dispersions in series $X$
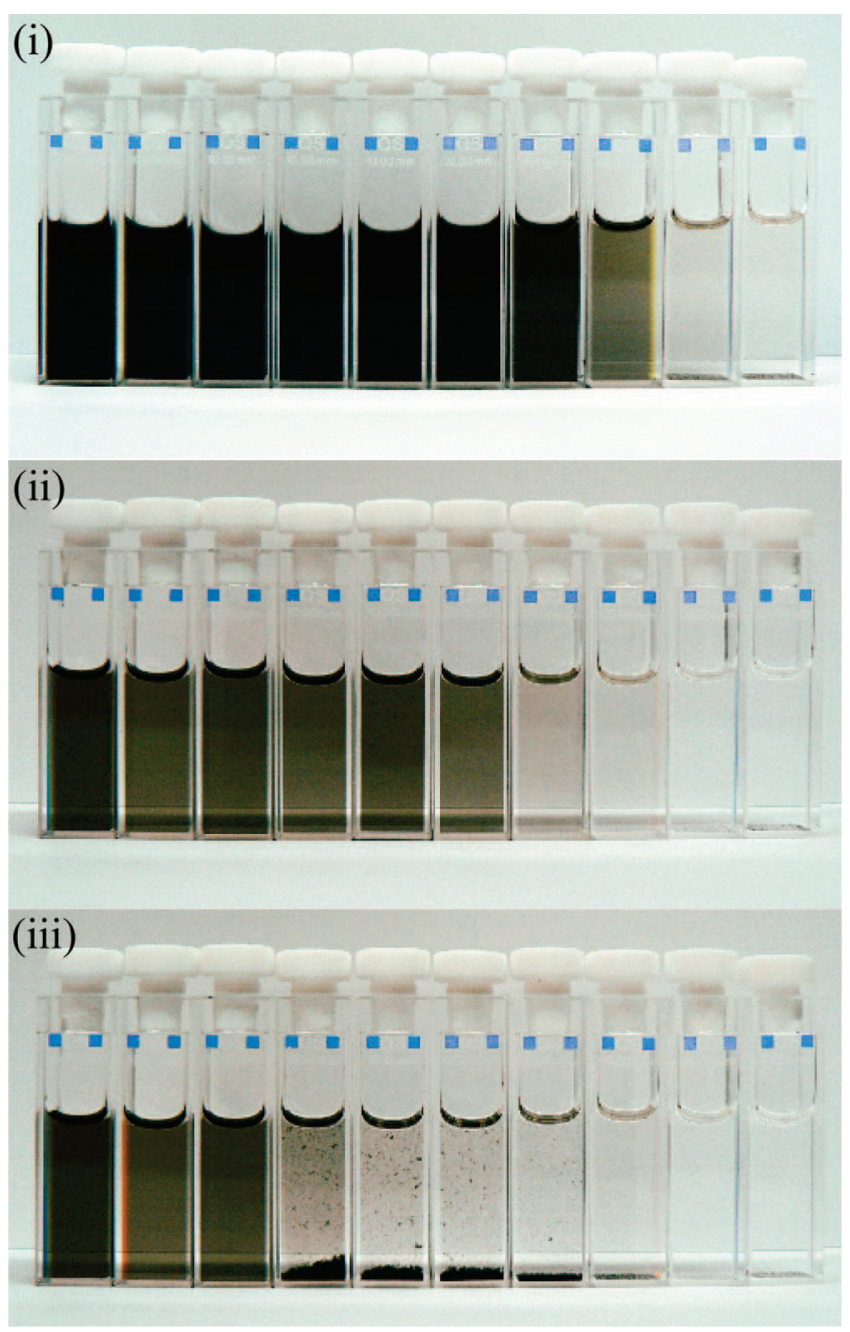

Figure 3. Photographs of SWNT dispersions prepared gradually reducing PVP concentration from left to right: (i) Original dispersion, series $X$; (ii) diluted dispersion $(12.5 \%)$ of series $X$, PVP concentration unchanged, series $Y$; (iii) diluted dispersion $(12.5 \%$ ) of series $X$, PVP concentration is $12.5 \%$ of series $X$ and $Y$, series $Z$. SWNT aggregation signifies the effect of PVP concentration on dispersion stability. See Table 1 and Figure 2 for actual values of PVP and SWNT concentration.

and $Y$. Here, SWNTs form aggregates at $\mathrm{PVP}_{\text {conc }} \approx 2.63 \mathrm{~g} / \mathrm{L}$ and below (see Figure 3 and Table 1). $\mathrm{PVP}_{\text {conc }}$ is therefore crucial to prevent reaggregation of the dispersed SWNTs. Our data suggest that a minimum of $\sim 3 \mathrm{~g} / \mathrm{L}(\sim 0.1 \mathrm{mM}) \mathrm{PVP}_{\text {conc }}$ is required to stabilize SWNT dispersions. Also, this effect does not necessarily depend on the $\mathrm{SWNT}_{\text {conc }}$ used in our experiments. High loading of SWNTs or stability of dispersions cannot be achieved with as-grown HiPco SWNTs. Indeed, the acid treatment during SWNT purification processes introduces $\mathrm{O}-\mathrm{H}$ groups on SWNT sidewalls. ${ }^{18,45,46}$ We speculate that the physical adsorption of PVP molecules on SWNT sidewalls in NMP relies strongly on the interaction between the oxidized $\mathrm{O}-\mathrm{H}$ groups on as-received purified HiPco SWNTs with the negatively charged carbonyl groups of the PVP molecules, ${ }^{47,48}$ unlike the aqueous SWNT dispersions by PVP. ${ }^{7}$ These negatively charged carbonyl groups arise due to the resonance structure of the pyrene rings in PVP molecules. ${ }^{48}$ In Section 3.3, we will argue that the dispersion mechanism is selective to SWNTs with diameters of $\sim 1 \mathrm{~nm}$. We attribute this to the inability of the PVP molecules to wrap around the small diameter SWNTs in spite of the abovementioned interaction. 

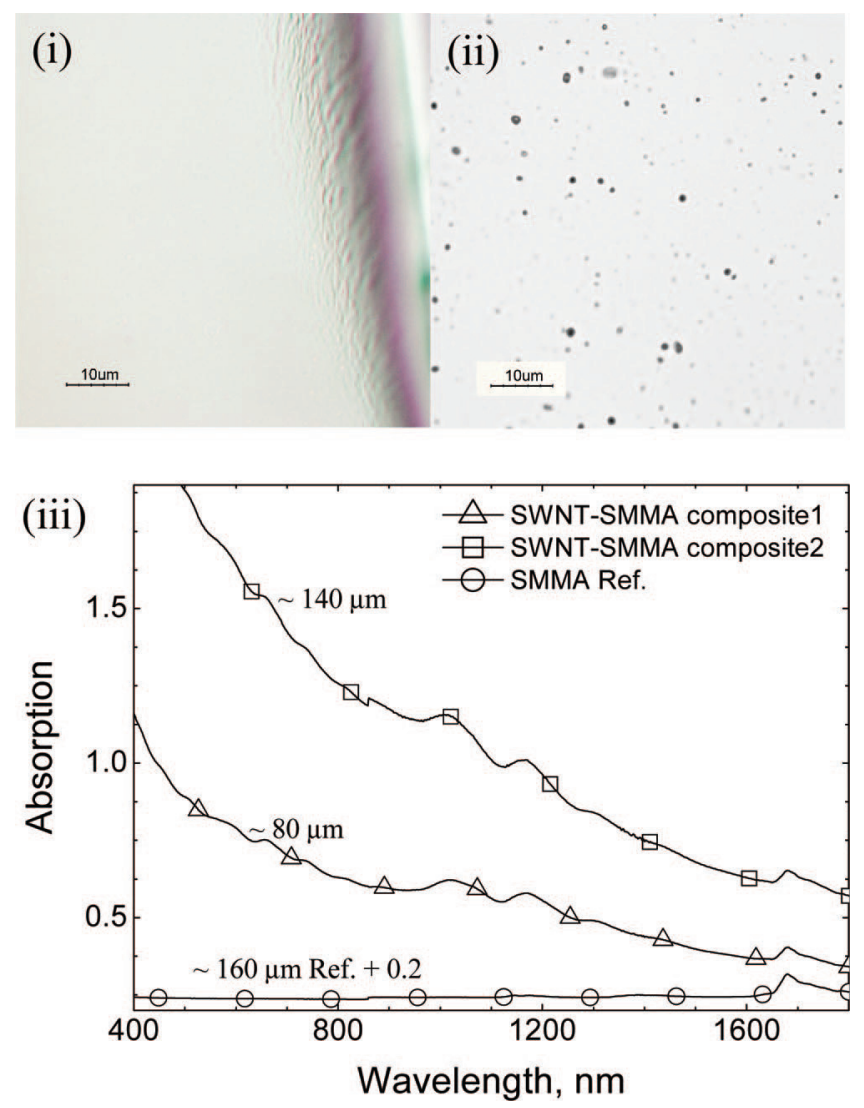

Figure 4. Optical micrograph of (i) SWNT-SMMA composite. Apart from the scribed area, the composite is completely featureless, with no SWNT aggregation. (ii) SWNT-SMMA composite prepared with $\sim 0.1$ $\mathrm{g} / \mathrm{L} \mathrm{SWNT}_{\text {conc }}$ showing $1-3 \mu \mathrm{m}$ SWNT aggregates (black dots). (iii) Absorption spectra of SWNT-polymer composites and of SMMA-PVP reference.

3.2. SWNT-SMMA Polymer Composites. We select SMMA as the host matrix for SWNT-polymer composites aimed at optical applications, because of its low sensitivity to humidity and good transparency below $\sim 1650 \mathrm{~nm}$, as seen from Figure 4(iii). E.g., this makes SMMA particularly attractive for the telecommunications $C$ band $(1530-1565 \mathrm{~nm})$. To avoid scattering losses, defects in the composites, such as cracks or voids and aggregated nanotubes must have much smaller dimensions than the device operation wavelength. ${ }^{30}$ Figure 4(i) is an optical micrograph of a representative SWNT-polymer composite prepared from sample $A$. Note that the film is intentionally scribed on the right side, in order to highlight the otherwise featureless surface. No SWNT aggregation is detected in the composite under an optical microscope, indicating the suitability of this composite for optical applications. The formation of SWNT bundles is a processing issue and is usually optimized by trial and error. Our data suggest that SWNT aggregation strongly depends on $\mathrm{SWNT}_{\text {conc }}$ and the viscosity of the polymer-SWNT dispersion mixture. Reducing the viscosity, or increasing $\mathrm{SWNT}_{\text {conc }}$, results in microscopic aggregations. We observe $\sim 3 \mu \mathrm{m}$ aggregates when $\mathrm{SWNT}_{\text {conc }}$ exceeds $\sim 0.1 \mathrm{~g} / \mathrm{L}$, as shown in Figure 4(ii). The absorption spectra of two SWNT-SMMA composites from sample $A$ without microscopic SWNT aggregations and pure SMMA films are presented in Figure 4(iii). The different level of optical density is achieved by varying the relative concentration of SMMA and SWNTs. No PL is observed from the composites, suggesting formation of sub-micrometer-sized SWNT aggregations. This is another important requirement for SWNT-polymer

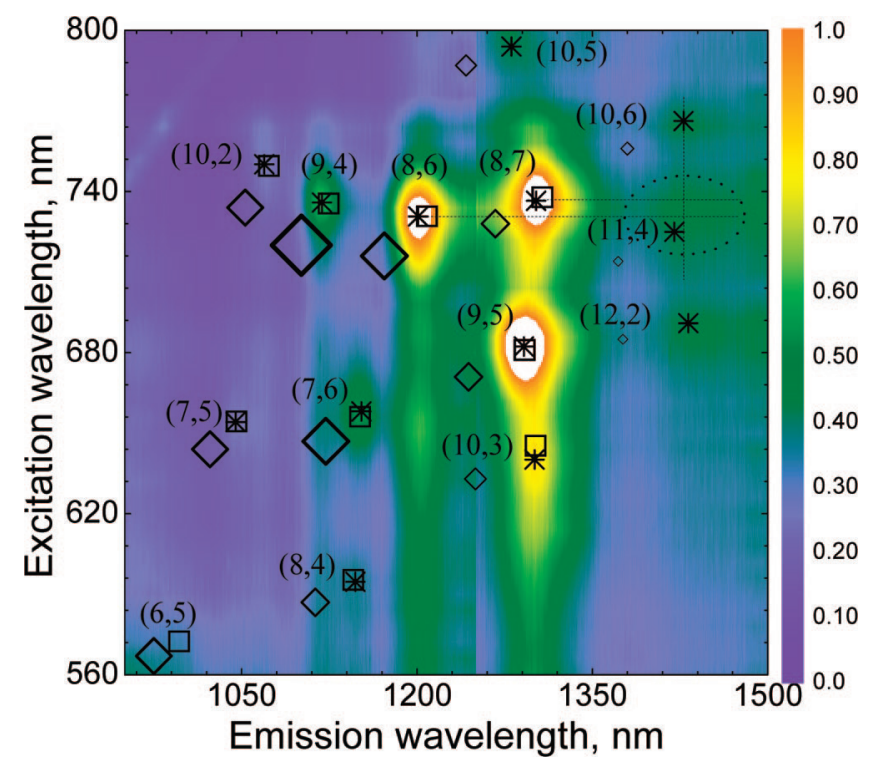

Figure 5. PLE map from isolated SWNTs dispersed in NMP and PVP, sample A1. The position of diamonds, squares and stars denote corresponding $\left(e h_{22}, e h_{11}\right)$ resonance spots from SWNTs in $\mathrm{D}_{2} \mathrm{O}-\mathrm{SDBS}$, pure NMP, and NMP-PVP, respectively. The size of the diamonds represents relative PL intensity of different nanotube species in $\mathrm{D}_{2} \mathrm{O}-$ SDBS. Features relating to exciton energy transfer appear at the cross sections of the horizontal $\left(e h_{22}\right.$ of $(8,6)$ and $\left.(8,7)\right)$ and vertical dashed lines $\left(e h_{11}\right.$ of $\left.(10,6)\right)$.

composite-based devices (e.g., saturable absorbers operating at $1550 \mathrm{~nm}^{27,49}$ ) since submicrometer bundling could increase the device speed, due to faster carrier relaxation, while avoiding unwanted scattering losses..$^{30,49}$

3.3. Isolated SWNTs in NMP-PVP. PL due to exciton recombination is expected from semiconducting SWNTs (s-SWNTs). ${ }^{6,44,50,51} \mathrm{PL}$ is not observed in large SWNT bundles. Exciton relaxation, via exciton energy transfer to s-SWNTs of larger diameters (i.e., smaller bandgaps ${ }^{52}$ ) in SWNT bundles is also possible. ${ }^{43,44,53}$ Therefore, high PL intensities from SWNT dispersions signify the presence of isolated SWNTs or small bundles. ${ }^{43,44}$ We observe PL from the vacuum-filtered PVP-aided SWNT dispersions in NMP. However, as with SWNTs dispersed in pure NMP ${ }^{11}$ these contain small SWNT bundles in addition to isolated ones. The big SWNT bundles can be removed from the dispersions by ultracentrifugation, enriching the supernatant solution with isolated SWNTs or small bundles (sample Al). This minimizes bundling-induced dielectric screening effects on the electronic properties of SWNTs..$^{23,41,42,44,54}$

Figure 5 plots the PLE map from sample $A 1$. The $\left(e h_{22}, e h_{11}\right)$ resonances from HiPco SWNTs in NMP-PVP (stars) are 20-40 meV red-shifted compared to those from SWNTs in $\mathrm{D}_{2} \mathrm{O}-\mathrm{SDBS}$ (open diamonds), but match reasonably well those from SWNTs in pure NMP of ref 33 (open squares). Reference 33 reported the presence of up to $\sim 70 \%$ isolated nanotubes by atomic force microscopy (AFM) measurement of deposits from a SWNT dispersion $(\sim 0.004 \mathrm{~g} / \mathrm{L})$ in pure NMP. ${ }^{33}$ Here we detect a small 2-6 meV blue-shift in sample $A 1$, as compared to ref 33. We attribute this to the smaller average bundle size $\mathrm{s}^{41,42,44}$ in sample $A 1$ compared to that in pure NMP, ${ }^{33}$ since dielectric screening due to PVP molecules causes a red-shift in the SWNT transition energies (compare samples $A$ and $D$ in Figure 1). The large red-shift in NMP or NMP-PVP in comparison to $\mathrm{D}_{2} \mathrm{O}-$ SDBS is due to dielectric screening by the solvent molecules. ${ }^{24-26} \mathrm{We}$ observe some weak features at $\sim(1428$, 732.6), (1432.7, 690), and (1428, 766) nm. In NMP-PVP dispersion, if we consider $\sim 15-30 \mathrm{meV}$ red-shift for the $(11,4)$, 


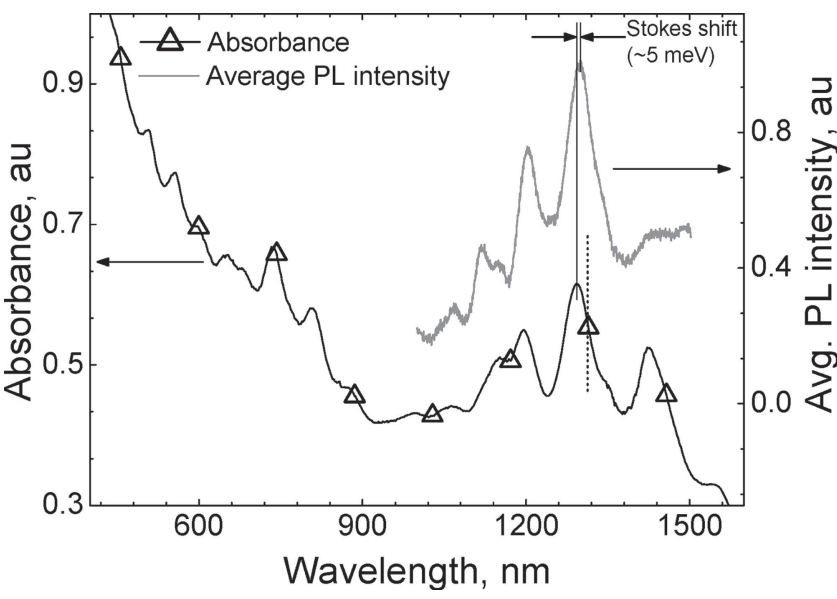

Figure 6. Absorption spectrum and average PL signal (excitation range 560-800 nm) from SWNTs in sample A1. Close resemblance between the two plots signifies mostly isolated SWNTs. The vertical dashed line shows the corresponding peak position in sample $A$.

$(12,2)$ and $(10,6)$ SWNTs compared to the $\mathrm{D}_{2} \mathrm{O}-\mathrm{SDBS}$ dispersion, then we can associate the weak features at $(1420,725)$, $(1432,690)$, and $(1428,766) \mathrm{nm}$ to the $\left(e h_{22}, e h_{11}\right)$ resonances from the $(11,4),(12,2)$, and $(10,6)$ species. The broad feature at $\sim(1427,732) \mathrm{nm}$ can be best described if we consider EET from the $(8,6)$ and $(8,7)$ species to the $(10,6),(11,4)$ and $(12$, 2) species. ${ }^{43,44,53}$ This is validated by the good alignment of the abovementioned broad feature with the $e h_{11}$ of $(10,6),(11,4)$, $(12,2)$ and $e h_{22}$ of $(8,6)$ and $(8,7)$. Recently, emission satellites associated with deep excitonic (DE) states have been observed in the PL spectra of isolated nanotubes. ${ }^{55}$ The DE features are usually much weaker than the main $e h_{11}$ PL emission peaks. Also, relative to the main $e h_{11} \mathrm{PL}$ emission peaks, the DE features are red-shifted by $\sim 40$ and $\sim 130 \mathrm{meV}$. However, in Figure 5 , the features indicated by an ellipse are red-shifted by $\sim 166$ and $\sim 85 \mathrm{meV}$ from the $e h_{11}$ of the $(8,7)$ and $(8,6)$ tubes, respectively. Therefore, we do not assign the broad and weak feature at $\sim(1427,732) \mathrm{nm}$ to the DE of $(8,7)$ and $(8,6)$.

In Figure 5, the size of the open diamonds represents the corresponding emission intensities in the $\mathrm{D}_{2} \mathrm{O}-\mathrm{SDBS}$ solution, showing a broad distribution of species of the dispersed SWNTs. A similar distribution is observed in SWNT dispersion in pure NMP. ${ }^{33}$ However, in sample A1, PL intensities from tubes with a diameter range of $0.84-0.92 \mathrm{~nm}$ are very weak. On the other hand, strong PL intensity from the $(8,6),(8,7)$, and $(9,5)$ tubes is observed, indicating preferential dispersion of SWNTs with a diameter range of $0.97-1.03 \mathrm{~nm}$. This observation is similar to our recent report where nanotubes with $\sim 1 \mathrm{~nm}$ diameter were spontaneously 'debundled' more readily than those with smaller diameter by the PVP molecules. ${ }^{11}$ Since PL intensity from smaller diameter SWNTs is stronger or comparable to that of the large diameter SWNTs, ${ }^{56,57}$ it can be concluded that after the PVP-aided dispersion, the relative population of the $(8,6)$, $(8,7)$, and $(9,5)$ tubes increases compared to the smaller diameter tubes e.g., $(9,4),(7,5),(7,6)$, and $(10,2)$, due to the inefficient dispersion of the latter group.

Comparison between the absorption spectrum and averaged PL signal (excitation range $560-800 \mathrm{~nm}$ ) of sample $A l$ is shown in Figure 6. The small red-shift of $\sim 5 \mathrm{meV}$ at about $1 \mathrm{eV}$ in the PL data compared to the absorption spectrum is due to Stokes shift. The Stokes shift between absorption and luminescence spectra can be associated with several factors: spectral shift due to the different systems for the two measurements, energy transfer occurring within bundles, ${ }^{44}$ and trap states due to structural or chemical defects. ${ }^{58}$ Similar values have been observed from SWNTs dispersed in aqueous media ${ }^{6,59,60}$ and from isolated SWNTs ${ }^{58}$ almost independent of the experimental setups. The EET signal from nanotube bundles is very weak for the present dispersion. Therefore, the $\sim 5 \mathrm{meV}$ red-shift observed here is not related to the experimental error or energy transfer. We assume that this small Stokes shift is the intrinsic value for isolated nanotubes due to the presence of trap states. Indeed, such trap states have been considered to understand the optical properties of isolated SWNTs. ${ }^{61}$ The blue-shift of $\sim 12$ $\mathrm{meV}$ at about $1 \mathrm{eV}$ (vertical dashed line) compared to the spectra of sample $A$ in Figure 1 is due to elimination of most SWNT bundles ${ }^{6,42,44}$ after ultracentrifugation. In this case, absorption is contributed by both bundled and isolated SWNTs, while only isolated s-SWNTs exhibit PL. Therefore, the strong similarity between the absorption spectrum and averaged PL signal in the $1000-1375 \mathrm{~nm}$ range indicates that most of the SWNTs in sample $A 1$ are isolated. ${ }^{6,60} \mathrm{AFM}$ measurements in ref 33 revealed the presence of $\sim 70 \%$ isolated SWNTs in pure NMP. ${ }^{33}$ As discussed before, the comparison of spectroscopic data strongly indicates that the average bundle size in sample $A l$ is smaller than that reported in ref 33 . The absorption peak at $\sim 1430 \mathrm{~nm}$ is strong. The corresponding peak in the averaged PL signal is very weak. As mentioned above, we assign most of the PL signal at $\sim 1430 \mathrm{~nm}$ to EET, ${ }^{43,44,53}$ since the $\left(e h_{22}, e h_{11}\right)$ resonances at $\sim 1430 \mathrm{~nm}$ emission in Figure 5 are too weak to be clearly resolved. Even if we conservatively assume the PL efficiencies of $(10,6),(12,2)$ and $(11,4)$ tubes to be comparable to that of $(8,7),(8,6)$, and $(9,5),{ }^{56,57}$ the population of the former group is expected to be very low. Thus, in Figure 6, the absorption band at $\sim 1430 \mathrm{~nm}$ due to these SWNTs should not be so strong. We therefore, assign the band at $\sim 1430 \mathrm{~nm}$ to an $\mathrm{O}-\mathrm{H}$ overtone, ${ }^{31,40}$ not to a nanotube exciton resonance. This also explains why this band in Figure 1 (vertical solid line) is not sensitive to the dielectric screening of the dispersant molecules. These dispersion procedures could be extended to graphite and graphene flakes. ${ }^{62}$

\section{Conclusions}

Dispersion of mostly isolated SWNTs with up to $\sim 0.16 \mathrm{~g} / \mathrm{L}$ can be achieved in NMP using PVP. This corresponds to $\sim 45$ wt $\%$ of the starting SWNT. Approximately $0.1 \mathrm{mM}$ of PVP is required to stabilize such solutions. In addition to high concentration, the dispersions contain a significant population of isolated SWNTs or small bundles, as evident from PL spectroscopy. SWNT-SMMA composites prepared from such dispersions show no aggregation by optical microscopy, making them ideal for photonics applications. Lastly, by PLE spectroscopy, we showed that PVP-aided SWNT dispersion in NMP, in addition to being efficient, is also sensitive to nanotube diameter.

Acknowledgment. The authors acknowledge funding from EPSRC grants No. GR/S97613/01 and EP/E500935/1, the Ministry of Information and Communication, Republic of Korea, Project No. A1100-0602-0101, the Royal Society Brian Mercer Award for Innovation, the European Reseach Council grant NANOPOTS, the Leverhulme Trust, the Newton Trust, the British Council/Ministero dell'Università e della Ricerca, T.H. from Schlumberger Cambridge Research Ltd. and Cambridge Commonwealth Trust. P.H.T. thanks the funding from NSF of China (No. 60521001). 


\section{References and Notes}

(1) Thess, A.; Lee, R.; Nikolaev, P.; Dai, H.; Petit, P.; Robert, J.; Xu, C.; Lee, Y. H.; Kim, S. G.; Rinzler, A. G.; Colbert, D. T.; Scuseria, G. E.; Tománek, D.; Fischer, J. E.; Smalley, R. E. Science 1996, 273, 483.

(2) Journet, C.; Maser, W. K.; Bernier, P.; Loiseau, A.; Lamy de la Chapelle, M.; Lefrant, S.; Deniard, P.; Lee, R.; Fischer, J. E. Nature 1997, 388,756 .

(3) Tersoff, J.; Ruoff, R. S. Phys. Rev. Lett. 1994, 73, 676.

(4) Girifalco, L. A.; Hodak, M.; Lee, R. S. Phys. Rev. B 2000, 62, 13104.

(5) Hertel, T.; Walkup, R. E.; Avouris, P. Phys. Rev. B 1998, 58, 13870.

(6) O'Connell, M. J.; Bachilo, S. M.; Huffman, C. B.; Moore, V. C.; Strano, M. S.; Haroz, E. H.; Rialon, K. L.; Boul, P. J.; Noon, W. H.; Kittrell, C.; Ma, J.; Hauge, R. H.; Weisman, R. B.; Smalley, R. E. Science 2002, 297, 593.

(7) O'Connell, M. J.; Boul, P.; Ericson, L. M.; Huffman, C.; Wang, Y.; Haroz, E.; Kuper, C.; Tour, J.; Ausman, K. D.; Smalley, R. E. Chem. Phys. Lett. 2001, 342, 265

(8) Moore, V. C.; Strano, M. S.; Haroz, E. H.; Hauge, R. H.; Smalley, R. E. Nano Lett. 2003, 3, 1379 .

(9) Haggenmueller, R.; Rahatekar, S. S.; Fagan, J. A.; Chun, J. H.; Becker, M. L.; Naik, R. R.; Krauss, T.; Carlson, L.; Kadla, J. F.; Trulove, P. C.; Fox, D. F.; DeLong, H. C.; Fang, Z. C.; Kelley, S. O.; Gilman, J. W. Langmuir 2008, 24, 5070.

(10) Zheng, M.; Jagota, A.; Semke, E. D.; Diner, B. A.; Mclean, R. S.; Lustig, S. R.; Richardson, R. E.; Tassi, N. G. Nat. Mater. 2003, 2, 338.

(11) Hasan, T.; Scardaci, V.; Tan, P. H.; Rozhin, A. G.; Milne, W. I.; Ferrari, A. C. J. Phys. Chem. C 2007, 111, 12594.

(12) Hasan, T.; Scardaci, V.; Tan, P. H.; Rozhin, A. G.; Milne, W. I.; Ferrari, A. C. Physica E 2008, 40, 2414.

(13) Maeda, Y.; Kimura, S.-i.; Hirashima, Y.; Kanda, M.; Lian, Y.; Wakahara, T.; Akasaka, T.; Hasegawa, T.; Tokumoto, H.; Shimizu, T.; Kataura, H.; Miyauchi, Y.; Maruyama, S.; Kobayashi, K.; Nagase, S. J. Phys. Chem. B 2004, 108, 18395

(14) Bahr, J. L.; Yang, J.; Kosynkin, D. V.; Bronikowski, M. J.; Smalley, R. E.; Tour, J. M. J. Am. Chem. Soc. 2001, 123, 6536.

(15) Chen, J.; Rao, A. M.; Lyuksyutov, S.; Itkis, M. E.; Hamon, M. A.; Hu, H.; Cohn, R. W.; Eklund, P. C.; Colbert, D. T.; Smalley, R. E.; Haddon, R. C. J. Phys. Chem. B 2001, 105, 2525.

(16) Yu, A.; Hu, H.; Bekyarova, E.; Itkis, M. E.; Gao, J.; Zhao, B. Haddon, R. C. Compos. Sci. Technol. 2006, 66, 1187.

(17) Viswanathan, G.; Chakrapani, N.; Yang, H. C.; Wei, B. Q.; Chung, H. S.; Cho, K. W.; Ryu, C. Y.; Ajayan, P. M. J. Am. Chem. Soc. 2003 125,9258 .

7. 17

(18) Banerjee, S.; Hemraj-Benny, T.; Wong, S. S. Adv. Mater. 2005 ,

(19) Britz, D. A.; Khlobystov, A. N. Chem. Soc. Rev. 2006, 35, 637.

(20) Bahr, J. L.; Tour, J. M. J. Mater. Chem. 2002, 12, 1952.

(21) Perebeinos, V.; Tersoff, J.; Avouris, P. Phys. Rev. Lett. 2004, 92 257402

(22) Finnie, P.; Homma, Y.; Lefebvre, J. Phys. Rev. Lett. 2005, 94 247401

(23) Wang, F.; Sfeir, M. Y.; Huang, L.; Huang, X. M. H.; Wu, Y.; Kim, J.; Hone, J.; O'Brien, S.; E., B. L.; Heinz, T. F. Phys. Rev. Lett. 2006, 96, 167401.

(24) Walsh, A. G.; Vamivakas, A. N.; Yin, Y.; Ünlü, M. S.; Goldberg, B. B.; Swan, A. K.; Cronin, S. B. Nano Lett. 2007, 7, 1485.

(25) Miyauchi, Y.; Saito, R.; Sato, K.; Ohno, Y.; Iwasaki, S.; Mizutani, T.; Jiang, J.; Maruyama, S. Chem. Phys. Lett. 2007, 442, 394.

(26) Ohno, Y.; Iwasaki, S.; Murakami, Y.; Kishimoto, S.; Maruyama, S.; Mizutani, T. Phys. Stat. Sol. B 2007, 244, 4002

(27) Rozhin, A. G.; Scardaci, V.; Wang, F.; Hennrich, F.; White, I. H.; Milne, W. I.; Ferrari, A. C. Phys. Stat. Sol. B 2006, 243, 3551.

(28) Chen, Y.-C.; Raravikar, N. R.; Schadler, L. S.; Ajayan, P. M.; Zhao, Y.-P.; Lu, T.-M.; Wang, G.-C.; Zhang, X.-C. Appl. Phys. Lett. 2002, 81 975
(29) Hertel, T.; Fasel, R.; Moos, G. Appl. Phys. A: Mater. Sci. Process. 2002, 75, 449 .

(30) Bohren, C. F.; Huffman, D. R. Absorption and Scattering of Light by Small Particles; Wiley-Interscience: New York, 1998.

(31) Ma, H.; Jen, A. K.-H.; Dalton, A. R. Adv. Mater. 2002, 14, 1339. (32) Set, S. Y.; Yaguchi, H.; Tanaka, Y.; Jablonski, M. J. Lightwave Technol. 2004, 22, 51.

(33) Giordani, S.; Bergin, S. D.; Nicolosi, V.; Lebedkin, S.; Kappes,

M. M.; Blau, W. J.; Coleman, J. N. J. Phys. Chem. B 2006, 110, 15708.

(34) Bahr, J. L.; Mickelson, E. T.; Bronikowski, M. J.; Smalley, R. E.; Tour, J. M. Chem. Commun. 2001, 193.

(35) Landi, B. J.; Ruf, H. J.; Worman, J. J.; Raffaelle, R. P. J. Phys. Chem. B 2004, 108, 17089.

(36) Shigeta, M.; Komatsu, M.; Nakashima, N. Chem. Phys. Lett. 2006, 418,115 .

(37) Wise, K. E.; Park, C.; Siochi, E. J.; Harrison, J. S. Chem. Phys. Lett. 2004, 391, 207.

(38) Liu, J.; Liu, T.; Kumar, S. Polymer 2005, 46, 3419.

(39) Nikolaev, P.; Bronikowski, M. J.; Bradley, R. K.; Rohmund, F.; Colbert, D. T.; Smith, K. A.; Smalley, R. E. Chem. Phys. Lett. 1999, 313, 91.

40) Chung, H.; Ku, M.-S. Vib. Spectrosc. 2003, 31, 125.

(41) O'Connell, M. J.; Sivaram, S.; Doorn, S. K. Phys. Rev. B 2004, $69,235415$.

(42) Reich, S.; Thomsen, C.; Ordejón, P. Phys. Rev. B 2002, 65, 155411.

(43) Tan, P. H.; Hasan, T.; Bonaccorso, F.; Scardaci, V.; Rozhin, A. G.;

Milne, W. I.; Ferrari, A. C. Physica E 2008, 40, 2352.

(44) Tan, P. H.; Rozhin, A. G.; Hasan, T.; Hu, P.; Scardaci, V.; Milne,

W. I.; Ferrari, A. C. Phys. Rev. Lett. 2007, 99, 137402.

(45) Hirsch, A. Angew. Chem., Int. Ed. 2002, 41, 1853.

(46) Hamon, M. A.; Hui, H.; Bhowmik, P.; Itkis, H. M. E.; Haddon, R. C. Appl. Phys. A: Mater. Sci. Process. 2002, 74, 333.

(47) Xu, W.; Chen, H.; Li, H.; Wang, M. Colloids Surf., A 2005, 266, 68.

(48) Pattanaik, M.; Bhaumik, S. K. Mater. Lett. 2000, 44, 352.

(49) Scardaci, V.; Sun, Z.; Wang, F.; Rozhin, A. G.; Hasan, T.; Hennrich,

F.; White, I. H.; Milne, W. I.; Ferrari, A. C. Adv. Mater. 2008, 20, 4040. (50) Bachilo, S. M.; Strano, M. S.; Kittrell, C.; Hauge, R. H.; Smalley, R. E.; Weisman, R. B. Science 2002, 298, 2361.

(51) Bachilo, S. M.; Balzano, L.; Herrera, J. E.; Pompeo, F.; Resasco,

D. E.; Weisman, R. B. J. Am. Chem. Soc. 2003, 125, 11186.

(52) Kataura, H.; Kumazawa, Y.; Maniwa, Y.; Umezu, I.; Suzuki, S.; Ohtsuka, Y.; Achiba, Y. Synth. Met. 1999, 103, 2555.

(53) Qian, H.; Georgi, C.; Anderson, N.; Green, A. A.; Hersam, M. C.; Novotny, L.; Hartschuh, A. Nano Lett. 2008, 8, 1363

(54) Fantini, C.; Jorio, A.; Souza, M.; Strano, M. S.; Dresselhaus, M. S.; Pimenta, M. A. Phys. Rev. Lett. 2004, 93, 147406.

(55) Kiowski, O.; Arnold, K.; Lebedkin, S.; Hennrich, F.; Kappes, M. M. Phys. Rev. Lett. 2007, 99, 237402

(56) Oyama, Y.; Saito, R.; Sato, K.; Jiang, J.; Samsonidze, G. G.; Gruneis, A.; Miyauchi, Y.; Maruyama, S.; Jorio, A.; Dresselhaus, G.; Dresselhaus, M. S. Carbon 2006, 44, 873 .

(57) Reich, S.; Thomsen, C.; Robertson, J. Phys. Rev. Lett. 2005, 95, 077402 .

(58) Berciaud, S.; Cognet, L.; Poulin, P.; Weisman, R. B.; Lounis, B. Nano Lett. 2007, 7, 1203.

(59) Hagen, A.; Moos, G.; Talalaev, V.; Hertel, T. Appl. Phys. A: Mater. Sci. Process. 2004, 78, 1137.

(60) Jones, M.; Engtrakul, C.; Metzger, W. K.; Ellingson, R. J.; Nozik, A. J.; Heben, M. J.; Rumbles, G. Phys. Rev. B 2005, 71, 115426.

(61) Hagen, A.; Steiner, M.; Raschke, M. B.; Lienau, C.; Hertel, T.; Qian, H. H.; Meixner, A. J.; Hartschuh, A. Phys. Rev. Lett. 2005, 95, 197401.

(62) Hernandez, Y.; Nicolosi, V.; Lotya, M.; Blighe, F. M.; Sun, Z.; De, S.; Mcgovern, I. T.; Holland, B.; Byrne, M.; Gunko, Y.; Boland, J.; Niraj, P.; Duesberg, G.; Krishnamurthy, S.; Goodhue, R.; Hutchison, J.; Scardaci, V.; Ferrari, A. C.; Coleman, J. N. Nat. Nanotech. 2008, 3, 563.

JP807036W 